\title{
Different options for prenatal testing for Huntington's disease using DNA probes
}

\author{
M FAHY*, C ROBBINS*, M BLOCH*, R W TURNELL†, AND M R HAYDEN* \\ From * the Department of Medical Genetics, University of British Columbia, Vancouver, Canada V6T 2B5; \\ and Regina Obstetrics and Gynecology, Saskatchewan, Canada S4P $3 X 3$.
}

SUMMARY The discovery of DNA markers closely linked to the gene for Huntington's disease (HD) has allowed development of predictive and prenatal testing programmes for HD. This report describes four different approaches to prenatal testing for $\mathrm{HD}$ which have arisen during a pilot predictive and prenatal testing program in British Columbia, Canada. In the first approach (exclusion testing), the at risk parent cannot or prefers not to learn of his/her HD status. Two other approaches involve definitive testing of a fetus when a parent is determined to be at increased risk to have inherited the HD gene or is affected with Huntington's disease. The fourth approach is a stepwise combination of the above two methods which we refer to as 'exclusiondefinitive' testing. These different approaches introduce a variety of challenging counselling and ethical issues. The role of each approach to prenatal testing in the management of Huntington's disease awaits the results of this and other predictive and prenatal testing programmes.

Huntington's disease is an inherited autosomal dominant neuropsychiatric disorder that usually manifests in mid-life with abnormal involuntary movement, personality change, and dementia. Although the cause of the disease is unknown, coordinated efforts have mapped the mutant gene to the distal end of the short arm of chromosome $4 .^{1-4}$ The recent discovery of a number of linked polymorphic DNA markers for HD has made it possible to determine whether subjects at risk are likely to have or have not inherited the mutant gene. ${ }^{4}$

The pilot programme for predictive and prenatal testing for HD began in Vancouver in November 1986. This is a research programme designed to assess the short and long term psychosocial effects of predictive and prenatal testing for HD. Preliminary results indicate that approximately $75 \%$ of candidates presenting for predictive testing can be given a modified risk estimate of having inherited the HD gene. ${ }^{5}$ To date (July 1988), 101 candidates have entered the Vancouver programme, six of whom have requested prenatal testing.

As the pilot programme develops and expands, the variety of clinical case situations increases and new options for testing are appreciated. With respect to prenatal testing for $\mathrm{HD}$, there are at present four approaches that differ depending on

Received for publication 22 August 1988.

Revised version accepted for publication 30 December 1988 . information known about or requested by the at risk parent. The first option is for an at risk parent who does not wish to learn of his/her own HD status but would like to know whether the fetus is at a very low risk or at the same risk as the at risk parent. Further, this may be the only prenatal option for some candidates where DNA is unavailable from crucial family members. It is referred to as 'exclusion' prenatal testing and such an approach has been reported previously by our group and others. ${ }^{67}$

The second situation occurs when an at risk parent participating in a predictive testing counselling programme is found to be at high risk for having inherited the HD gene. Prenatal testing is then available to determine whether or not the fetus is likely to have inherited the HD gene. This would be considered 'definitive' prenatal testing. Such an example has not been reported to date but is likely to occur in the near future, when predictive testing programme participants found to be at high risk wish to have children with a low risk of having inherited the HD gene.

The third approach has not previously been described. It is a stepwise combination of exclusion prenatal testing and predictive testing. The first step of the prenatal test would be exclusion testing. If the fetus were found to be at low risk, the at risk parent could continue to remain uninformed concerning his/her own risk and presumably complete the 
pregnancy. However, if the fetus was found to have a risk similar to that of the at risk parent (that is, close to $50 \%$ risk) the next step would be to determine definitively the phase of the marker segregating with $\mathrm{HD}$. In this way the status of the parent at risk, and hence the genetic status of the fetus relative to HD, is also determined. With this approach, termination of a fetus unlikely to have inherited the HD gene would be avoided (that is, the $50 \%$ outcome for the simple exclusion testing).

The final situation occurs when the candidate either presents with early signs or symptoms of HD and is clinically diagnosed as affected or has previously been diagnosed as having HD. When this occurs, definitive prenatal testing is available to determine whether the fetus is at high risk.

We report here the use of linked DNA markers for prenatal testing for $\mathrm{HD}$ in the latter two situations.

\section{Case reports}

CASE 1

The first situation involved a proband who is a 28 year old, at risk woman anxious to have children. She had become familiar with the predictive testing programme and was unsure as to whether she wished to pursue such testing on her own behalf, but was most concerned about the possibility of passing on the HD gene to future offspring. The exclusion testing approach had been discussed with her but was rejected as she did not wish to terminate a pregnancy at $50 \%$ risk.

The proband and her partner requested a counselling session to discuss prenatal options. She proposed the following approach as an alternative to predictive testing for herself and exclusion testing for the fetus.

(1) Perform exclusion testing, that is, determine whether or not the fetus has inherited a chromosome 4 from the affected grandparent, without determining what form of the marker is segregating with the HD gene.

If the fetus has not inherited a chromosome from the affected grandparent, it would be at low risk. No further testing would be requested (fig 1, outcome A). If the fetus has inherited a chromosome from the affected grandparent the fetus would be at approximately $50 \%$ risk, which is equal and linked to the status of the at risk parent (fig 1, outcome B).

(2) In the event of a $50 \%$ (approximately) fetal risk outcome, she wished to proceed to determine which form of the marker segregates with the gene for HD. This will determine whether or not both the at risk parent and the fetus are truly at high risk or alternatively at low risk. Thus, the two possible

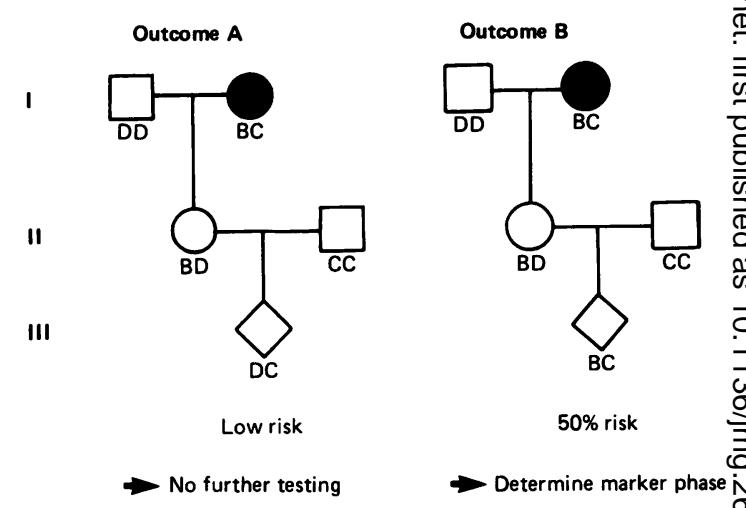

FIG 1 The stepwise approach to prenatal testing. If the fetus has a low risk of having $H D$, no further testing is performed. If the fetus has a risk similar to the parent at $50 \%$ risk the phase of the marker segregating with $H D$ is determined.

outcomes of the definitive testing step are that both $\vec{\oplus}$ the fetus and the at risk parent will be found to be at. high risk or both at low risk of having inherited the gene for HD.

The proband proposed this approach because of her preference not to have her own risk determined by DNA analysis unless doing so might avert $\stackrel{\square}{\square}$ termination of a $50 \%$ at risk fetus. She and her $\overrightarrow{\vec{P}}$ partner are presently reviewing their options to $\frac{\hat{3}}{3}$ decide on an approach most suitable for them. If, $\vec{\partial}$ after careful explanation and counselling considering the implications of this decision, the parents request this approach, this will be offered in the context of a pilot research programme for predictive ${ }^{\circ}$ and prenatal testing for $\mathrm{HD}$.

CASE 2

The definitive testing approach was carried out for $\mathrm{a}_{\mathrm{O}}$ woman recently diagnosed as having HD. The proband is the 22 year old daughter of a 48 year. oldo woman at present suffering from HD. The proband's maternal grandfather was also affected with $N$ HD and died aged 44. A grandpaternal uncle and N three grandpaternal first cousins were similarly affected and died in their early 40s.

The proband is the youngest in a sibship of five, none of whom has yet shown signs of HD. She was? noted to have exhibited symptoms as early as $18_{\mathrm{C}}^{\mathbb{\Phi}}$ years of age. Clinical diagnosis was based on classical presentation including choreiform movements, restlessness, and slurred speech. CT scan

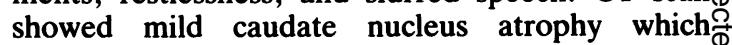
together with a positive family history confirmed the 2 diagnosis of $\mathrm{HD}$. 
Based on the confirmed diagnosis of HD and the risk to future offspring, the proband, at present in a stable relationship, was considering tubal ligation to avoid future pregnancies. At this time she was not using any mechanical or oral contraceptive. A pregnancy test was performed and was determined to be positive. A therapeutic abortion was considered as she did not wish to risk transmitting this gene to her offspring. She was informed that prenatal testing was possible and, after careful consideration, she stated that the outcome would influence her decision concerning the pregnancy. She and her partner wished to continue the pregnancy if they could be reassured that the fetus was at low risk.

The predictive prenatal test was administered within the guidelines for prenatal testing for HD offered by the University of British Columbia. The counselling programme involves a total of three pretest sessions of approximately one hour each (including counselling for the CVS prenatal procedure), a delivery of results session, and six follow up sessions or phone contact dates (according to the outcome of the prenatal procedure) over a two year period.

\section{DNA studies}

DNA was extracted from the blood of the proband, her partner, and the affected maternal grandmother (to the fetus) to determine informativeness of the test for prenatal testing, as previously described. ${ }^{6}$ The DNA was digested with HindIII, BglI, and $T a q \mathrm{I}$, electrophoresed, blotted, and hybridised with subclones of D4S10 and D4S95. The results illustrated that the prenatal test would be informative (fig 2).

A chorionic villus sample was obtained by transcervical aspiration with ultrasound guidance during the ninth week of gestation. A $50 \mathrm{mg}$ specimen was obtained. DNA was extracted from the fetal tissue and digested with HindIII, BgII, and TaqI, electrophoresed, blotted, and hybridised with pk082, R7 (subclones of D4S10), and D4S95, respectively.

\section{Results}

In this family it was possible to distinguish clearly which form of the marker segregated with the HD gene (fig 2). From the affected maternal grandmother, the proband inherited allele $A$ detected after HindIII digestion, allele B detected after $B g I I$ digestion, and allele $\mathrm{A}$ detected after TaqI digestion from the affected maternal grandmother. Thus, the haplotype ABA is predicted to be linked to the HD gene with at least $96 \%$ accuracy. The proband

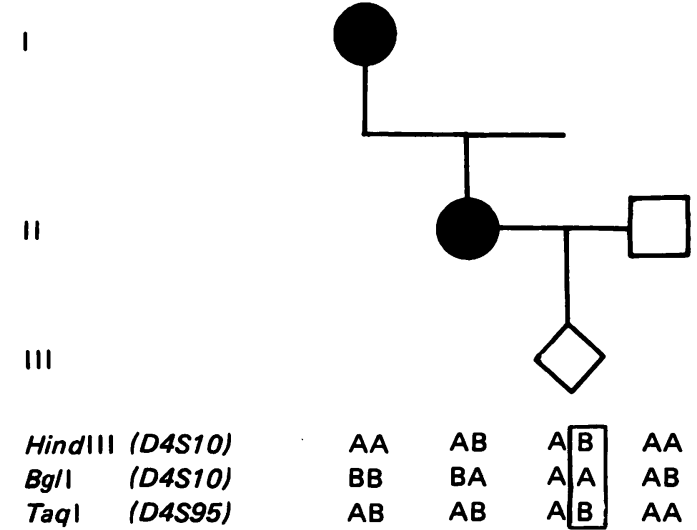

FIG 2 The fetus has inherited the $B A B$ haplotype from the affected parent. This confers a very low risk for the fetus of having inherited the gene for $H D$.

inherited the haplotype BAB from the unaffected maternal grandfather.

The fetus was determined to have inherited the BAB or maternal grandfather's haplotype from the affected mother (fig 2). This implies that the fetus is at very low risk $(<4 \%)$ of having inherited the HD gene. On the basis of this information the parents decided to continue the pregnancy.

\section{Discussion}

The use of polymorphic DNA markers linked to the locus for Huntington's disease has made predictive and prenatal testing possible. Using three DNA markers (D4S10, D4S62, and D4S95) and a total of seven enzymes (HincII-D4S62, EcoRI, BglI, HindIII-D4S10, AccI, TaqI, MboI-D4S95), none of 50 controls was found to be homozygous for all the markers. The informativeness of such a test is now only limited by the availability of DNA from affected and unaffected family members and the recombination fraction between the linked markers and the HD locus. ${ }^{5}$

The case situation, which we refer to as exclusiondefinitive testing, enables the $50 \%$ at risk parent to maximise the a priori $75 \%$ probability that the fetus has not inherited the HD gene. The $50 \%$ probability that the fetus is at very low risk by virtue of not having the affected maternal grandmother's chromosome is either confirmed or ruled out by the exclusion testing step. In the event that the fetus is determined to have inherited the affected grandmother's chromosome, the phase of the markers segregating with the gene for HD is determined. This approach to prenatal testing was suggested by a 
subject at risk who would, if at all possible, ideally prefer not to learn of her status, but would request information about her own risk rather than terminate a $50 \%$ at risk fetus. Thus, such a person anticipates that an 'uncertain' termination (that is, at $50 \%$ risk) will be more emotionally devastating than learning of an increased risk for herself.

There are certain concerns inherent in an approach of this nature. Until this time, the UBC predictive testing programme has suggested that a subject at risk should have predictive testing before a pregnancy if they wish to have definitive prenatal testing. For a person who has not learned of his/her HD status before a pregnancy, either because of insufficient time or uncertainty as to whether or not to have the test, exclusion prenatal testing has formerly been the only option offered. This policy is based on concern for the emotional and psychological status of the subject at risk after having to learn of a possibly increased risk for themselves and the fetus. In this worst case scenario, the at risk person would learn that he/she is at a high risk, as is the fetus, and yet still have to deal with the emotional trauma associated with terminating a pregnancy. However, for some, the stepwise approach may be worthy of serious consideration, especially for those who were considering predictive testing before the pregnancy.

Exclusion-definitive testing also confers an additional risk to the fetus as a result of the prenatal diagnostic procedure itself. Of course, if predictive testing were performed before pregnancy, prenatal diagnosis would only be an option for those persons at risk who were given an increased likelihood of having inherited the gene. Notwithstanding this slightly increased risk of miscarriage to the fetus $(\approx 1 \%)$, the exclusion-definitive stepwise approach may be a more preferable route for some couples at risk.

The second case situation is an example of the most informative prenatal test where definitive information concerning the at risk parent's status was already available (that is, the mother was clinically affected). Furthermore, it was possible to determine which particular form of the marker segregated with the gene for HD using DNA available from the crucial family members, in particular the affected maternal grandmother. Consequently, it was possible to provide the affected mother with information critical to her decision regarding procreation.

A potential issue regarding definitive testing is that this approach may be suitable only for persons with early HD, as informed consent may be difficult to obtain once the disease has progressed. For this couple, they mutually decided not to terminate the pregnancy as they planned together to care for the baby with the realisation that increasing responsibil- $\stackrel{\vec{\Phi}}{\rightarrow}$ ity for raising the child will fall on the unaffected $\underset{\vec{D}}{\vec{D}}$ parent as the disease progresses.

It is apparent that options for prenatal testing are varied and clearly more complex than initially $\frac{\bar{\sigma}}{\bar{s}}$. suspected. An appropriate choice is dependent upon $\frac{\vec{\sigma}}{\vec{\sigma}}$ how much information candidates wish to learn $\propto$ about themselves or the fetus or both and what is technically possible. The decision making process $\overrightarrow{0}$ incorporates the parents' values, such as the importance of having children and attitudes towards $\vec{\omega}$ termination, as well as their feelings about their perceived ability to cope with the stress of learning of an increased risk and their hopes for treatment is for HD in the future. A detailed clinical assessment of of the couple's ability to cope with this information $\omega$ is necessary. In the end, however, the candidate and $\mathrm{W}$ his/her partner choose an option based on their 을 current needs, tolerance for risk factors, and perceived impact of the various possible outcomes. $\subseteq$

The precise role of each of these approaches to prenatal testing for HD awaits the long term follow $\vec{\bullet}$ up of this and other predictive testing programmes following detailed and in depth analysis. In the exclusion-definitive stepwise approach, the proband 8 and her partner are prepared to continue a pregnancy if they can be reassured that the fetus is at low risk, $\frac{\partial}{0}$ at the expense of possibly having to learn of the at $\stackrel{0}{\not}$ risk partner's status. For the mother recently $\stackrel{\square}{\unrhd}$ diagnosed as having $\mathrm{HD}$, prenatal testing enabled $\overrightarrow{\overrightarrow{\vec{O}}}$ her to have a child determined to be at very low risk $\frac{0}{3}$ for $\mathrm{HD}$. At least one major concern, that is, $\overrightarrow{\vec{T}}$ transmitting the mutant gene to her offspring, has been lifted for this affected person.

We would like to thank Dr Oksana Suchowersky and Mary Lou Klimek of the Department of 3 Medical Genetics, Alberta Children's Hospital, Calgary, for CVS counselling and procedures. The $\frac{\circ}{3}$ support of the Woodward Foundation, the Huntington Society of Canada, NHRDP, and other private donations are acknowledged.

\section{References}

${ }^{1}$ Gusella JF, Wexler NS, Conneally PM, et al. A polymorphic $\stackrel{N}{\Omega}$ DNA marker genetically linked to Huntington disease. Nature N 1983;306:234-9.

2 Hayden MR, Hewitt J, Wasmuth JJ, et al. A polymorphic DNA N marker that represents a conserved expressed sequence in the $\sigma$ region of Huntington disease gene. Am J Hum Genet 1988;42: 125-31.

3 Wasmuth JJ, Hewitt J, Smith B, Allard D, et al. A highly polymorphic locus very tightly linked to the Huntington's ? disease gene. Nature 1988;332:734-6.

4 Meissen GJ, Meyers RH, Mastromauro CA, et al. Predictive $\frac{-}{0}$ testing for Huntington's disease with the use of linked DNA $\mathbb{\mathbb { D }}$ marker. N Engl J Med 1988;318:535-42.

5 Hayden MR, Robbins C, Allard D, et al. Improved predictive testing for Huntington's disease using three linked DNA markers. Am J Hum Genet 1988;43:689-94. 
${ }^{6}$ Hayden MR, Hewitt J, Kastelein JJ, et al. First trimester prenatal diagnosis for Huntington's disease with DNA probes. Lancet 1987; i:1284-5.

7 Quarrell OW, Meredith AL, Tyler K, et al. Exclusion testing for Huntington's disease in pregnancy with a closely linked DNA marker. Lancet 1987;i:1281-3.
Correspondence to Dr Michael R Hayden, Department of Medical Genetics, F168 University Hospital, 2211 Wesbrook Mall, Vancouver, BC V6T 2B5, Canada. 